\title{
Supporting a Mobile Lost and Found Community
}

\author{
Dominique Guinard \\ Auto-ID Labs, ETH Zurich \\ Sonneggstrasse 63 \\ $\mathrm{CH}-8092$ Zurich, Switzerland \\ +41588717846 \\ dguinard@ethz.ch
}

\author{
Oliver Baecker \\ I-Lab, University of St. Gallen \\ Dufourstrasse 40a \\ CH-9000 St. Gallen, Switzerland \\ +41712247720 \\ oliver.baecker@unisg.ch
}

\author{
Florian Michahelles \\ Auto-ID Labs, ETH Zurich \\ Sonneggstrasse 63 \\ $\mathrm{CH}-8092$ Zurich, Switzerland \\ +41446327477 \\ fmichahelles@ethz.ch
}

\begin{abstract}
In the era of ubiquitous devices and mobility, we increasingly carry objects of great value (in terms of data, money or emotions). Because of our increased mobility, we are also more inclined to lose these objects. When it comes to finding them again, current lost property offices seem rather inflexible and not fully adapted to our nomad lives. They lack dynamic information, introduce too many intermediates and induce high costs. We support the growth of a community of users able to solve the problem on their own using their mobile phones. We describe our concept and implementation of the idea based on prototypes of mobile phones enhanced with a novel type of RFID (Radio Frequency IDentifiaction) reader, the use of the EPC (Electronic Product Code) standards and the creation of both mobile and server-side software. We finally discuss how it can help making the current system more dynamic and efficient.
\end{abstract}

\section{Categories and Subject Descriptors}

D.3.3 [Information Systems Interfaces and Presentation]: Multimedia Information Systems - augmented realities.

\section{General Terms}

Design, Economics, Human Factors.

\section{Keywords}

Lost and Found, Mobile Interactions, Internet of Things, RFID.

\section{INTRODUCTION}

Losing something of great emotional or intrinsic (money or data!) value is often a shock. In this kind of situations we currently rely on lost property offices implemented and run by the travel business (airlines, train companies, coach services, etc.) or governmental organizations. In a pre-study undertaken by our group [1] it was identified that more than $400^{\circ} 000$ items were lost in Switzerland in the year 2006. Amongst these less than $40 \%$ were recovered.

In an era of high mobility, the solutions we rely on suffer from a number of problems. On the one hand, they lack dynamic information and compatibilities amongst the systems; on the other hand, they involve many intermediates and have high costs and no revenues for the institutions running them.

We believe the traditional approach to retrieve lost items can be enhanced by reducing the intermediates making it a more community-oriented process where finders are directly linked to

Permission to make digital or hard copies of all or part of this work for personal or classroom use is granted without fee provided that copies are not made or distributed for profit or commercial advantage and that copies bear this notice and the full citation on the first page. To copy otherwise, or republish, to post on servers or to redistribute to lists, requires prior specific permission and/or a fee.

MobileHCI 2008, September 2-5, 2008, Amsterdam, the Netherlands. Copyright (C) 2008 ACM 978-1-59593-952-4/08/09...\$5.00. owners. This improves the chances of recovery, simplifies the system and lowers costs [1]. Combined with the use of mobile phones and RFID tags, this can improve the dynamic information available to the owner. For example, a consultant can know whether he simply left his laptop at home or whether it is lost and the incident needs to be reported to his company.

In our lost and found system, called EPCFind, we create a simple Internet of (your) Things [2], with which we interact using mobile devices to help us tracing and recovering our belongings while on the run. In more concrete terms, with the "Distributed Tracing" approach (see Section 2.2), we help the owner (Bob) getting dynamic information about where his laptop might be located and in the "Community-Based Reporting" (see Subsection 2.1) we help the finder (Alice) easily reporting the recovery of Bob's laptop while being on the move and without the need for intermediates.

\section{CONCEPT}

As for a number of systems, the existence of intermediates in the traditional approach decreases the efficiency and increases the costs. This fact was confirmed by interviews with experts at the Swiss National Railways (SBB), which run a large share of the Swiss lost property offices. Ideally, when Alice finds Bob's laptop, she should be able to report it directly to Bob. Of course this approach is not new and people have been enabling this direct link for years using address tags providing contact details. This idea is rather straightforward but implies a number of problems: (i) First of all, it reveals the owner's identity to everyone able to read the tag. (ii) Secondly, it suffers from updates: every time you change your address you need to change every name tag. (iii) Thirdly, it denatures the object you tag by adding a relatively big label to it (it is rather unlikely that iPhone owners would tag their beloved device with $5 \times 5 \mathrm{~cm}$ address tags). (iv) Finally, there might be no real incentive for the finder to return the found object (see Section 4.2). While Section 2.1 describes the communitybased reporting of a discovered item, we discuss the distributed tracing of lost items in Section 2.2.

\subsection{Community-Based Reporting}

To tackle the discussed problems, we propose to support a community of mobile phone users, which are able to communicate directly with the owners whenever they find an object. For this purpose, we use the EPCFind mobile software and wireless technologies. When Alice finds Bob's laptop, she can easily and quickly report the recovery by scanning the tag on the object. In order to do so, she uses the Report application of EPCFind, which connects to a central server and finds out about the object's owner (fulfilling ii). If Alice accepts it, the mobile application creates a trace of the recovery and reports it to Bob. Note that the system does not have to reveal Bob's identity. Instead, Bob uses the 
application (see Figure 1) to directly contact Alice and arranges a way of sending the laptop back (ensures i).
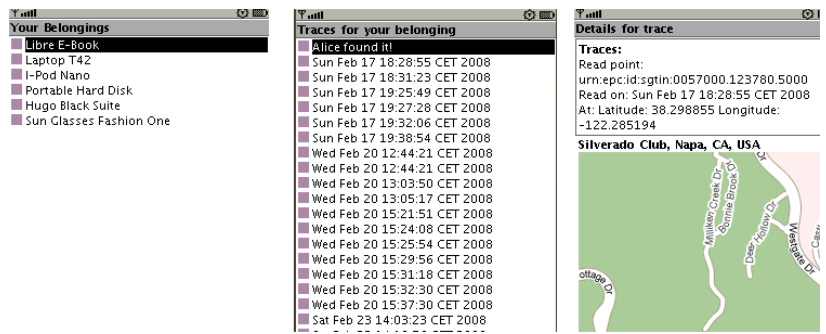

Figure 1: Owner's Mobile User Interface

\subsection{Distributed Tracing}

Community-based reporting fulfills the need for eliminating intermediates and eases the reporting process: Alice does not need to find the next lost property office and she can directly report the recovery to Bob using the EPCFind system. Yet, the system in this state does not resolve Bob's need for dynamic information: what if Bob's laptop was still at home? What if it got stolen and not simply lost? What if no one found it?

To solve this issue, we extend our system with a distributed network of readers made available by the community. As proposed by Frank et al. [8], we assume a network of readers formed by static (e.g. readers already in place in stores) and mobile devices (e.g. an RFID-enabled mobile phone). These distributed readers can silently (i.e. without explicit human interaction) register tagged objects in their vicinity. With EPCFind, Bob can use the application on his mobile phone to locate where his laptop was last "seen" by the distributed readers and make an appropriate decision based on this information (e.g. call the police, call his home, report the loss of his laptop to the company, etc.).

Similarly, the silent reporting can be used in order for Bob to register the presence of its own objects next to his mobile phone on a regular basis (e.g. while the phone and the laptop are on his desk at home, etc.). This approach reduces the privacy concerns inherent to the Distributed Tracing approach while not filtering the most valuable information in our case: when was the object last seen next to Bob?

\section{IMPLEMENTATION}

\subsection{Selecting Physical Objects}

Core to our system is the notion of "selecting" or "scanning" physical objects using a mobile phone. The subject has been explored by several researchers already. In [6] Rukzio et al. identified touch as being a well-received interaction technique.

Using NFC (Near Field Communication) seems quite natural for our application. However, because of its very limited range (i.e. touch), NFC needs a visible tag or zone of interaction. Beyond denaturing the object, it also concentrates the interaction metaphor on the tag rather than on the object.

We would like to change the interaction paradigm from identifying a tag representing the object, to identifying the object itself. This, we believe, can make the system rather easy and straightforward to use. Thus, we decided to explore the use of EPC tags. These tags, based on the EPC standard (see Subsection 3.2) are using UHF (Ultra High Frequency), which means they can be read from a greater distance. Hence, using these tags, EPCFind users can select an object simply by touching it anywhere they would like with the reader.

\subsection{Leveraging the EPC Network}

The potential of the EPCFind system is relying on the size and contribution of the community. In more technical terms, a critical mass of mobile phones with RFID readers and tagged objects is required. Furthermore, the tagged objects need to disclose a number, which enables the unique identification of the object, unlike barcodes, which identify a type of product. Finally, as mentioned before, the reading range of the mobile readers needs to be greater than touch distance (ideally $20-30 \mathrm{~cm}$ ).

We propose using UHF tags, implementing the EPC Gen2 (Electronic Product Code tags, second generation ${ }^{1}$ ) standard. These RF transponders fulfill both the need for a world-wide unique, instance-level ID and a greater read range. Furthermore, the planned deployment of the code on retail products (e.g. Walmart in the US or Metro in Europe) would prevent from having to put tags on objects manually as they would already be tagged. It also permits to assign the ownership of an object at purchase time: freeing Bob from both having to tag his objects and registering them as his belongings. Indeed, by matching the EPC on the laptop with a unique number identifying Bob (e.g. his phone number, loyalty / credit card number, etc.), we can assign the ownership at the store, when Bob buys his laptop.

The only drawback of this approach is the lack of UHF readers embedded in commercial mobile phones [2]. In order to overcome this problem for the implementation of EPCFind, we use three prototypes of Nokia E61i mobile phones (Figure 2). These phones are equipped with UHF EPC RFID reader as a functional cover (i.e., the reader is integrated into the phone battery cover) potentially capable of reading up to $30-50 \mathrm{~cm}$. From an HCI viewpoint this is quite interesting improvement over NFC mobile phones, since it slightly changes the way objects are identified.

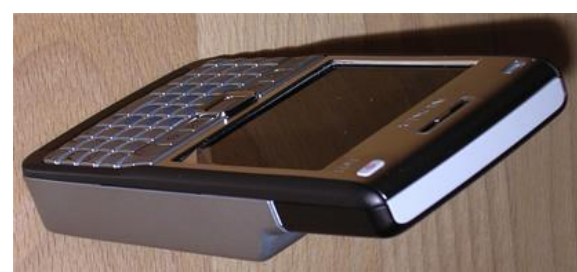

Figure 2: Nokia E61i enhanced with an EPC RFID Reader.

Besides providing a way to encode unique numbers on tags (EPC Tag Standard), and addressing the reading of UHF tags (Reader Standard), the EPC Network standards also offer a way of capturing and querying EPC events. This is done in the EPC Information Server (EPCIS) $)^{2}$, which is the basis for our implementation of the EPCFind backend.

\subsection{Implementing the EPCFind Software}

\subsubsection{Mobile Software}

The EPCFind software consists of two parts: the first one is the user interface implemented in Java Mobile Edition (CLDC), which needs to be installed on the mobile device of every member

\footnotetext{
${ }^{1}$ http://www.epcglobalinc.org/

${ }^{2}$ http://www.accada.org/epcis/
} 
of the community. It is composed of three distinct MIDlets, each representing one part of the application. The Report MIDlet is used by Alice to report the recovery of Bob's laptop. It activates the UHF RFID reader on the mobile phone and asks Alice to approach the phone to the object in order to identify it. It then reports the recovery to the EPCFind backend using either a WiFi or a GPRS connection. The AutoReport MIDlet is a process, which can run in the background in order to implement the distributed tracing. It activates the reader and reports an RFID event to the EPCFind backend each time a (new) tag is in the scope of the reader. The Find MIDlet is the counterpart of the Report and AutoReport MIDlets. It enables Bob to retrieve information about his belongings as shown in Figure 1. Using a unified interface, it offers access to two types of information: traces and recoveries. Traces are the events generated silently by the AutoReport MIDlet. They provide information about where an object was last seen, and thus allow deducing where it might be located. Recoveries are generated whenever a member of the community uses the Report MIDlet to signal the recovery of an object to its owner.

\subsubsection{Server-side Software}

The aim of the EPCFind backend is mainly to act as an information server, storing the events reported by the mobile software (Report and AutoReport MIDlet) and providing an interface for queries (Find MIDlet). Thus, the EPCFind backend complies with the EPCIS standard. However, it adds two main functionalities: First of all, unlike the EPCIS, it needs to offer an interface that mobile devices, with limited resources, can interact with. Secondly, it needs to hold a notion of ownership, i.e. it needs to know what belongs to Bob and control the information Bob and Alice can access. As a consequence, we implemented the EPCFind backend as a Java Enterprise application interfacing with Accada, an open-source implementation of the EPCIS standard as shown in Figure 3.

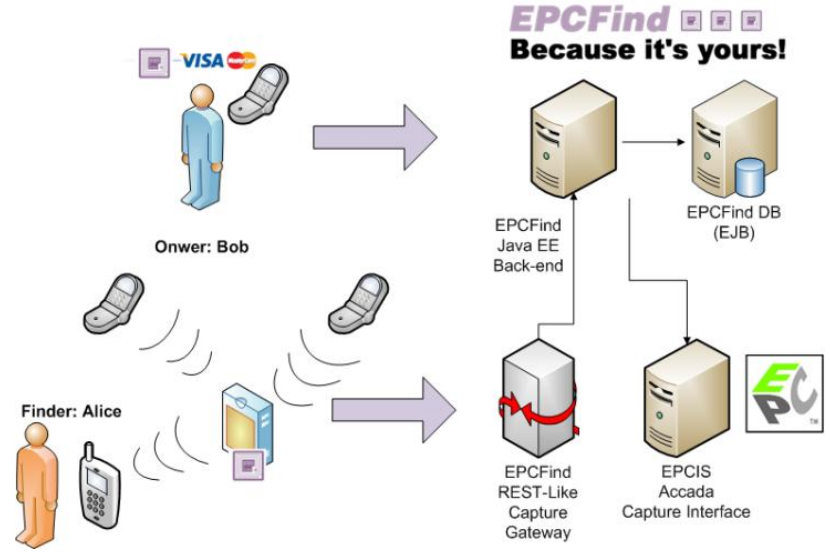

Figure 3: Interactions with the Server-side Software.

\section{DISCUSSION}

\subsection{Privacy Considerations}

As for a number of applications involving automated and pervasive tracking of objects or people, EPCFind raises some privacy issues. While, we do not pretend solving all of them with our current implementation let us briefly identify flaws and discuss solutions based on other works in the area.
First of all, because of the distributed tracing, the EPCFind backend contains location information about ones' belongings. This could potentially enable Alice to track Bob by querying the system for the location of his laptop. We take a rather simple approach and prevent this from happening by selectively giving access to object traces as hinted in [3]: A user can only query the system for traces of items he owns. Note that this method is not fail-proof and restricts quite a lot the possible usage of the precious data EPCFind collects. Kriplean et al. extensively discuss the general issue of protecting Auto-ID information servers in [3].

The other privacy concern is driven by the fact that users carry tagged objects, which can be read in a silent manner and from a distance. This introduces two major problems: Firstly, the EPC mobile phones could be used to "x-ray" bags or suitcases and detect items one does not want to publicly show. Secondly, the tags one carries on a regular basis could be used to profile the user (e.g. by stores) and possibly identify him using inference techniques and information leaks. These two flaws are not inherent to the EPCFind system, but rather to wireless systems (and beyond) and thus are explored extensively in literature. Partial solutions for RFID range from encrypting the tags to providing means for "killing" a tag, a solution supported by the EPC Gen2 tags [4].

\subsection{Business Perspective and Incentive}

From a business perspective, two main challenges need to be tackled to secure the success of EPCFind. Firstly, the EPCFind application needs to be hosted by a trusted third party, if users are expected to report privacy-critical tracing data. Secondly, it needs to be ensured that finders would actually report the recovery of valuable items such as laptops.

To cope with the first challenge, an insurance company can position itself as a trusted third party between owners and finders of objects. This approach stems from expert interviews we conducted with insurance managers. As insurance companies face the challenge of differentiating themselves from their competitors to grow in saturated markets, they seek for innovative services that are complimentary to their core business. In the case of EPCFind, they can offer a service to retrieve lost belongings and insure the remaining risk of not recovering them. Based on the conducted expert interviews, this concept has a large positive image effect for insurers and creates valuable additional contact points between the insurer and his customers. In addition, customers associate a high level of credibility with insurance companies, which addresses the identified first challenge. By integrating the reporting and finding functionality with their insurance portal, insurers can also generate additional advertisement-relevant traffic leading to cross-selling potential.

As a result of the conducted interviews, we know that insurance companies are not interested in hosting a central lost and found infrastructure, because they do not want to deal with the storage and return of lost objects. The proposed community-based approach deals with this concern by enabling a direct interaction between owner and finder. Besides the mentioned benefits, another major motivation for insurance companies to host an EPCFind solution is the access to detailed information about their customers. The EPC-based knowledge about a lost object enables them to conduct restitution in kind, which means the insurer replaces the item instead of compensating the customer for the purchase of a similar one. This allows controlling the restitution 
process and selecting an appropriate retailer leading to reduced costs for the insurer. The recovery of lost items is also of interest to insurers, because it potentially decreases cases of insurance fraud where lost objects are claimed as stolen. This leads to decreased costs from insurance claims as most insurance companies insure only the theft, but not the loss of objects.

To cope with the second challenge, the insurer could create incentives for using the EPCFind solution. An example is a reward for the finder, while the owner pays a fee to query for traces and recoveries (see Section 3.2). If the sum of paid fees exceeds the sum of issued rewards, the insurer can even crossfinance the operating costs for the EPCFind infrastructure.

\subsection{Formative Demonstration}

While no real user study of the prototype has been undertaken yet, we had the chance of having several people testing it during a demonstration [5]. On the positive side, people liked the feeling of scanning the object using the EPC mobile phone reader. The fact that they could select an object simply by touching it anywhere rather than having to find a tag or a barcode was well perceived. People also liked the community idea where one could directly return found items by interacting with a mobile phone.

However, they had two concerns about it. Firstly, they had doubts whether the finder would really return valuable items. This fact motivated our discussions with insurances as exposed in Subsection 4.2. Secondly, they were concerned about privacy, especially when demonstrating the Distributed Tracing approach. We are aware of this fact and the discussion in Subsection 4.1 could be used as a basis for designing an improved prototype in terms of privacy.

\section{RELATED WORK}

On top of the related work we mentioned throughout the previous parts of this paper, this section discusses inspiring research project working on similar applications as well as the state-of-the-art in lost and found solutions.

With over 22 million registered items Immobilize [9] is the world's largest personal property registration and recovery service. Users can register descriptions, photos and certificates of ownership of their items online, and the data can be used to recover the items or file insurance claims in case of theft or loss. It is possible to manually link RFID tags to items, but the product information still needs to be recorded via a web interface. EPCFind is leveraging the EPC Network and EPC tags instead of using custom solutions to register, trace, and recover items. Of course, the usefulness of this approach depends on the application of EPC tags for future products. While, the effectiveness of the Immobilize approach depends on the amount of users, EPCFind takes the community-based approach a step further by directly connecting owner and finder.

The CRUISE/r system [7], a combination of RFID and WLAN technology, is based on peoples' cooperation to allow for the tracing of lost objects. The traces are based on the IPv6 address of wireless access points that communicate to a user's mobile phone, which in turn uses its RFID reader to discover the personal belongings a user carries with him. To discover a lost object, the last access point that "saw" the object queries the mobile phones of close-by users, which in turn use their RFID readers to search for the object. Compared to EPCFind, the CRUISE/r system relies on existing infrastructure (namely the wireless access points), which is not likely to be available aside urban areas. In addition, it considers only the active query for the trace of a lost object, but not the active reporting of a discovered object. Finally, the novelty of the EPCFind approach lies in the application of mobile phones that are capable of reading UHF tags, which enable an increased read range.

In [8], Frank et al. present the architecture, design, and evaluation of an object search system that relies on sensor-enabled mobile phones to discover lost objects. The concept does not rely on a pre-installed infrastructure, but uses mobile phones as hubs to a ubiquitous infrastructure. The work focuses on heuristics to distribute a search query to a subset of users in order to reduce costs and time until an object is retrieved. While the authors used Bluetooth technology for their real-world experiments, they argue that any object tagging technology could be used with their system. Again, the proposed system focuses on the query for lost objects and not on the reporting of discovered items by a finder or the immediate notification of users by an underlying infrastructure.

\section{ACKNOWLEDGMENTS}

This project was supported by Nokia Research. Our thanks go to Sassan Iraji and his team at Nokia Research Helsinki for their great work on the Nokia E61i RFID prototype.

\section{REFERENCES}

[1] U. Maeder: " Smart Lost \& Found Services for Insurers", Master Thesis, University of St. Gallen and ETH Zurich, Switzerland, 2007. Abstract on http://ilab.ch/docs/Abstract_I-07.03SmartLost_Found.pdf

[2] T. Wiechert, F. Thiesse, F. Michahelles, P. Schmitt, E. Fleisch: "Connecting Mobile Phones to the Internet of Things: A Discussion of Compatibility Issues between EPC and NFC.", AMCIS '07, Keystone, Colorado, USA, 2007.

[3] T. Kriplean, E. Welbourne, N. Khoussainova, V. Rastogi, M. Balazinska, G. Borriello, T. Kohno, D. Suciu: "Physical Access Control for Captured RFID Data", IEEE Pervasive Computing,vol. 6, no. 4, pp. 48-55, 2007.

[4] A. Juels, "RFID Security and Privacy: A Research Survey", IEEE Journal of Selected Areas in Communications, vol. 24, pp. 381- 394, 2006.

[5] D. Guinard, F. Michahelles, S. Iraji, H. Hirvola, E. Fleisch: "EPCFind: Implementing a Mobile Lost and Found Infrastructure”, HotMobile '08, Napa Valley CA, USA, 2008.

[6] E. Rukzio, K. Leichtenstern, V. Callaghan, P. Holleis, A. Schmidt, J. Chin: "An Experimental Comparison of Physical Mobile Interaction Techniques: Touching, Pointing and Scanning”, Ubicomp '06, California, USA, 2006.

[7] Y. Tobe: "Converting Crowds of People to Advantage: Aplication to a Lost and Found System", in: Procedings of the Workshop on UbiComp in the Urban Frontier, held at UbiComp '04, Nottingham, UK 2004.

[8] C. Frank, P. Bollinger, C. Roduner, and W. Kellerer: "Objects Calling Home: Locating Objects Using Mobile Phones", in: Proceedings of Pervasive '07, Toronto, ON, Canada, 2007.

[9] https://www.immobilise.com/home.ik 